\title{
ALEITAMENTO MATERNO EXCLUSIVO: PREVALÊNCIA NA MATERNIDADE E DURANTE O SEGUNDO MÊS DE VIDA
}

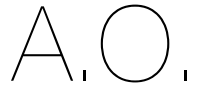

ARTIGO ORIGINAL

Serviço de Neonatologia, JAG da Mulher e da Criança do Centro

Hospitalar Universitário de São João,

Alameda Hernâni Monteiro, 4200-319 Porto, Portugal

*Endereço para correspondência:

Jorge Santos Silva Serviço de Neonatologia do Hospital São João, Alameda Hernâni Monteiro, 4200-319 Porto, Portugal jsantossilva@netcabo.pt

Histórico do artigo:

Recebido a 11 de novembro de 2020

Aceite 16 de marco de 2021

\section{BREASTFEEDING: PREVALENCE AND DETERMINING FACTORS AT MATERNITY DISCHARGE AND DURING THE SECOND MONTH OF LIFE}

Jorge Santos Silva ${ }^{1}$; Filipa Flor-de-Lima'; Henrique Soares ${ }^{1}$

RESUMO

INTRODUÇÃo: A taxa de aleitamento materno exclusivo dos recém-nascidos em Portugal mantém-se inferior às recomendações Nacionais e Internacionais.

OBJETIVOS: O objetivo deste trabalho foi determinar a prevalência do aleitamento materno exclusivo à alta da maternidade e a sua evolução até ao segundo mês de vida.

METODOLOGIA: Realizámos um estudo retrospetivo de recém-nascidos saudáveis nascidos no nosso hospital, de abril a novembro de 2019. Os dados referentes ao seu modo de alimentação foram recolhidos em consulta durante o segundo mês de vida. RESULTADOS: Dos 159 recém-nascidos estudados, o aleitamento materno exclusivo ocorreu em 80,5\% à alta da maternidade, sendo a sua prevalência inferior nos nascidos por cesariana $69,7 \%(p=0,019)$. Durante o segundo mês de vida a prevalência do aleitamento materno exclusivo ocorreu em $59,7 \%$ da amostra $(p<0,001)$ não se demonstrando nessa altura diferenças significativas entre os diferentes modos de parto.

DISCUSSÃo DOS RESULTADOS: Destaca-se a relação negativa do nascimento por cesariana na prevalência do aleitamento materno exclusivo à alta da maternidade. Realça-se a importância do acompanhamento à família após a alta hospitalar, assim como, o reforço da formação continua dos profissionais de saúde.

CONCLUSÕES: A prevalência do aleitamento materno exclusivo da nossa amostra, embora inferior ao preconizado pela literatura, é sobreponível aos dados nacionais. A sua melhoria é dependente de uma estratégia clínica diferenciada para os diferentes modos de nascimento, assim como, pela implementação da Iniciativa Hospital Amigo dos Bebés.

\section{PALAVRAS-CHAVE}

Aleitamento materno exclusivo, Maternidade, Recém-nascidos

ABSTRACT

INTRODUCTION: The Portuguese rate of exclusive breastfeeding is lower than recommended by National and International entities. OBJECTIVES: The aim of this study was to determine the prevalence of exclusive breastfeeding, at maternity discharge, and during the babies first two months of life.

METHODOLOGY: A retrospective study was done on a sample of healthy children born in our hospital from April to November 2019 Data regarding the babies feeding mode was obtained from parents at the babies outpatient visit.

RESULTS: Of a total of 159 babies studied, exclusive breastfeeding occurred in $80.5 \%$ at maternity discharge. This value decreased to $69.7 \%$ ( $p=0.019$ ) in those born by cesarean section. The prevalence of exclusive breastfeeding during the second month of life occurred in $59.7 \%$ of the sample $(p<0.001)$ although no significant difference was shown to occur for the different modes of birth. DISCUSSION OF RESULTS: Birth by cesarean section has a significant negative influence on exclusive breastfeeding at maternity discharge. Family support after maternity discharge is of primordial importance as is the continual staff training.

CONCLUSIONS: Although less prevalent than recommended, exclusive breastfeeding in our sample was in the published reference range for Portugal. It's improvement is dependent on a clinical strategy taking into account the different birth modes and the implementation of the Baby Friendly Hospital Initiative.

\section{KEYWORDS}

Exclusive breastfeeding, Maternity ward, Neonates 


\section{INTRODUÇÃo}

A alimentação com leite humano (LH) do recém-nascido (RN) é reconhecidamente a melhor forma de o alimentar, promovendo assim o seu melhor crescimento e desenvolvimento (1). A organização mundial da Saúde (OMS), recomenda o aleitamento materno exclusivo (AME), até aos 6 meses de idade, e depois o AM complementado com outros alimentos, até aos 2 anos de idade (1-3). As vantagens são múltiplas e documentadas, tanto no período neonatal, como na infância e futura vida adulta (1). A promoção do AME é assim um fator crucial de saúde pública e a sua implementação no período pós-natal imediato de primordial importância. É assim, também em Portugal, uma prioridade da Direção-Geral da Saúde (DGS) na sua global promoção de saúde pública $(4,5)$.

Apesar de todas as recomendações Nacionais $(4,5)$ e Internacionais (6) a prevalência do AME em Portugal mantém-se a níveis inferiores ao pretendido e com decréscimo acentuado nos primeiros meses de vida. A última publicação do Registo do Aleitamento Humano da DGS (RAM), de 2014 (7), refere AME de 92\% dos RN à alta da maternidade e uma posterior quebra para 68\% às 5-6 semanas de vida. De acordo com o Relatório de Resultados do Inquérito Alimentar Nacional e de Atividade física, de 2017 , 46\% das crianças foram amamentadas exclusivamente com leite humano durante um período inferior a 4 meses (8). A literatura demonstra dados semelhantes, apontando diversos fatores responsáveis pelo abandono precoce do AME (7-13).

\section{OBJETIVOS}

O nosso objetivo foi o de determinar a prevalência local do AME dos RN saudáveis à alta da maternidade, assim como a sua manutenção ao segundo mês de vida.

\section{METODOLOGIA}

Realizámos um estudo transversal, de RN nascidos de Abril a Novembro de 2019 num hospital terciário e urbano do Norte do País. Analisámos uma amostra de RN saudáveis de termo, não gemelares, com percurso perinatal sem intercorrências ou necessidades terapêuticas, observadas posteriormente, ao segundo mês de idade, na Consulta de Recém-nascidos (CRN). Estas CRNs ocorrem durante os vários dias da semana e o agendamento das crianças é aleatório e composto pela totalidade de RN com alta da maternidade. A amostra deste estudo é composta por RN seguidos em consulta pelo mesmo médico. Recolhemos dados clínicos dos respetivos processos do internamento e da consulta. Além do tipo de aleitamento realizado, registámos a idade materna, a idade gestacional, tipo de parto, género e peso do RN e se ocorreu abandono do AME. O conceito de AME foi definido como uma alimentação exclusiva de leite humano, sendo aceitável os suplementos vitamínicos, minerais ou tratamentos farmacológicos.

A análise estatística foi realizada através do programa SPSS ${ }^{\circledR}(\mathrm{IBM}$, USA) v.26. As variáveis contínuas foram caracterizadas pela média ( \pm desvio padrão) e as variáveis categóricas pelas frequências absoluta e relativa. A comparação de variáveis contínuas foi realizada através do Teste $t$ independente e as categóricas através do Teste quiquadrado. A análise emparelhada entre a data da alta e o segundo mês de vida foi realizada através do Teste McNemar. O valor $\mathrm{p}$ inferior a 0,05 foi considerado estatisticamente significativo.

Foi obtido autorização da Comissão de Ética do hospital.

\section{RESULTADOS}

Dos 159 RN avaliados, 87 (55\%) eram do género feminino, idade gestacional média de 39 semanas $\left(\mathrm{DP}_{ \pm} 1,2\right)$ e peso médio de $3173 \mathrm{~g}$ $(\mathrm{DP} \pm 1460 \mathrm{~g})$ ao nascimento. A idade média materna era de 31,8 anos $(\mathrm{DP} \pm 5,9)$. O parto foi eutócico em $82(51,6 \%)$ dos casos, por ventosa em $44(27,6 \%)$ e cesariana nos restantes $33(20,8 \%)$.

À alta da maternidade, o AME ocorreu em 128 (80,5\%) dos casos, sendo destes, 71 (55,5\%) nascidos por parto eutócico, 36 (28,1\%) por ventosa e $21(16,4 \%)$ por cesariana $(p=0,019)$.

A prevalência do AME diminuiu desde a alta da maternidade de $80,5 \%$ para $59,7 \%(p<0,001)$ no segundo mês de vida. Não se verificaram, no entanto, diferenças significativas na comparação do modo de parto e a prevalência do AME no segundo mês de vida (Tabela 1).

\section{Tabela 1}

Tipo de aleitamento à alta da maternidade e segundo mês de vida

\begin{tabular}{|c|c|c|c|c|c|c|}
\hline & & MATERNIDADE & & \multicolumn{3}{|c|}{ SEGUNDO MÊS DE VIDA } \\
\hline & $\begin{array}{c}\text { ALEITAMENTO } \\
\text { MATERNO EXCLUSIVO } \\
(\mathrm{n}=128)\end{array}$ & $\begin{array}{c}\text { ALEITAMENTO } \\
\text { MATERNO NÃO } \\
\text { EXCLUSIVO } \\
(n=31)\end{array}$ & VALOR $p$ & $\begin{array}{c}\text { ALEITAMENTO } \\
\text { MATERNO EXCLUSIVO } \\
2^{\circ} \text { MES DE VIDA } \\
(n=95)\end{array}$ & $\begin{array}{l}\text { ALEITAMENTO } \\
\text { MATERNO NÃO } \\
\text { EXCLUSIVO } \\
2^{\circ} \text { MÊS DE VIDA } \\
(\mathrm{n}=64)\end{array}$ & VALOR $p$ \\
\hline $\begin{array}{l}\text { Idade mãe, anos } \\
\text { média }( \pm D P)\end{array}$ & $31,4( \pm 5,7)$ & $33,3( \pm 6,6)$ & \multirow[t]{2}{*}{$0,115^{\star *}$} & $31,8( \pm 5,0)$ & $31,7( \pm 7,1)$ & \multirow[t]{2}{*}{$0,873^{\star \star}$} \\
\hline Mín-máx & $18-42$ & $18-43$ & & $18-42$ & $18-43$ & \\
\hline $\begin{array}{l}\text { Idade gestacional, semanas } \\
\text { média }( \pm D P)\end{array}$ & $39,1( \pm 1,2)$ & $38,7( \pm 1,3)$ & \multirow[t]{2}{*}{$0,113^{\star \star}$} & $39,1( \pm 1,2)$ & $38,8( \pm 1,2)$ & \multirow[t]{2}{*}{$0,134^{\star \star}$} \\
\hline Mín-máx & $36-41$ & $36-41$ & & $36-41$ & $36-41$ & \\
\hline $\begin{array}{l}\text { Peso, gramas } \\
\text { média }( \pm \mathrm{DP})\end{array}$ & $3164,6( \pm 371,9)$ & $3210,6( \pm 448,5)$ & \multirow[t]{2}{*}{$0,554^{\star *}$} & $3199,2( \pm 376,3)$ & $3135,5( \pm 402,1)$ & \multirow[t]{2}{*}{$0,310^{\star \star}$} \\
\hline Mín-máx & $2375-4245$ & $2365-4075$ & & $2375-4245$ & $2365-4075$ & \\
\hline \multicolumn{7}{|l|}{ Género, n (\%) } \\
\hline Feminino & $75(58,6)$ & $13(41,9)$ & \multirow{2}{*}{$0,094^{\star}$} & $55(57,9)$ & $33(51,6)$ & \multirow{2}{*}{$0,431^{*}$} \\
\hline Masculino & $53(41,4)$ & $18(58,1)$ & & $40(42,1)$ & $31(48,4)$ & \\
\hline \multicolumn{7}{|l|}{ Tipo parto, n (\%) } \\
\hline Eutócico & $71(55,5)$ & $11(35,5)$ & \multirow{3}{*}{$0,019^{*}$} & $53(55,8)$ & $29(45,3)$ & \multirow{3}{*}{$0,278^{\star}$} \\
\hline Ventosa & $36(28,1)$ & $8(25,8)$ & & $26(27,4)$ & $18(28,1)$ & \\
\hline Cesariana & $21(16,4)$ & $12(36,7)$ & & $16(16,8)$ & $17(26,6)$ & \\
\hline
\end{tabular}

* Teste qui-quadrado

${ }^{\star *}$ Teste $\mathrm{t}$ independente 
Na consulta, o peso médio das crianças era de $4351 \mathrm{~g}$ (DP \pm 738 g; 29206300) não tendo ocorrido um ganho ponderal médio significativamente diferente para os diferentes tipos de parto ou tipo de aleitamento ( $p=0,954$ ), ganho médio 1269g nos RN sob AME versus 1307 g nos RN sob AM não exclusivo.

O motivo referido para o abandono do AME foi por decisão parental em 9 dos 34 casos, sendo os restantes por indicação de profissionais de saúde por hipogalactea materna e má evolução ponderal. De salientar que num dos casos, um aleitamento misto (leite humano com fórmula infantil) realizado na maternidade, passou a AME posteriormente à alta hospitalar.

\section{DISCUSSÃO DOS RESULTADOS}

Analisando a prevalência do AME de $80,5 \%$ à alta da maternidade, constatámos um nível inferior ao preconizado por entidades de Saúde Nacionais e Internacionais, embora comparáveis aos dados nacionais publicados. Dos fatores comparados, a idade materna, a idade gestacional, o peso ao nascimento e o género do lactente, não demonstraram diferenças estatisticamente significativas do AME à alta, sendo, no entanto, significativa essa diferença na comparação do modo de parto, ocorrendo menos nos nascimentos por cesariana. Este dado poderá ter importância no estabelecimento privilegiado, neste grupo, de uma estratégia de orientação antecipatória da amamentação, especialmente na cesariana programada, assim como um apoio e orientação adicional às mães que amamentam, durante o primeiro dia pós parto e após a alta da maternidade (14-16).

Como esta amostra é composta por RN saudáveis, poderemos postular que a melhoria destes índices de AME seriam dependentes de melhorias assistenciais multifatoriais da nossa instituição, nomeadamente nas áreas Obstétrica e Neonatal. Propomos, assim, a evolução assistencial preconizada e estabelecida na Iniciativa Hospital Amigo dos Bebés (1720). Para que um hospital sejam nomeado Hospital Amigo dos Bebés terá que cumprir as dez medidas preconizadas nessa Iniciativa sendo assim expectável uma melhoria dos índices neonatais de AME da nossa instituição (19).

Esta melhoria da prevalência de AME também será esperada nas primeiras semanas de vida em consequência da implementação desta iniciativa assim como em resposta a um possível programa domiciliar, personalizado, de apoio ao aleitamento materno a ser direcionado especificamente à primeira semana pós-parto, pois ocorreu como demonstrado uma quebra de $26 \%$ após a alta da maternidade.

\section{CONCLUSÕES}

Este estudo de RN saudáveis demonstrou a diminuição da prevalência do AME entre a alta da maternidade e a avaliação ao segundo mês de vida. Salienta-se que a abordagem institucional não pode ser a mesma para todos os grupos, havendo dificuldades acrescidas em alguns, nomeadamente nos nascidos de forma distócica.

O sucesso do AME passará pela implementação de cuidados clínicos de acordo com o preconizado pela Iniciativa Hospital amigo dos Bebés assim como na possível instituição de um programa de apoio domiciliar durante a primeira semana após a alta da maternidade. A formação continua e envolvimento de todos os profissionais de saúde são a essência do programa.

\section{REFERÊNCIAS BIBLIOGRÁFICAS}

1. Horta BL, Victoria CG. Long-term efects of breastfeeding. A systemic review. Geneva: World Health Organization; 2013. https://apps. who.int/iris/handle/10665/79198.

2. The optimal duration on exclusive breastfeeding-Report of an expert consultation. Geneva: World Health Organization; 2001.
3. World health Organization. The Global Strategy for Infant and Young Child Feeding. Geneva: WHO; 2003

4. Programa Nacional de Saúde Infantil e Juvenil. Lisboa: Direcção Geral da Saúde; 2013.

5. Alimentação Saudável dos 0 aos 6 anos. Linhas de orientação para profissionais e educadores. Lisboa: Direção Geral de Saúde; 2019.

6. European Union Project on Promotion of Breastfeeding in Europe. Protection, promotion and support of breastfeeding in Europe: a blueprint for action. Luxembourg: European Commission, Directorate Public Health and Risk Assessment; 2004.

7. Orfão A, Santos C, Magalhães C. Registo do aleitamento humano. Relatório Janeiro a Dezembro de 2012. Lisboa: Direção Geral da Saúde;2013.

8. Lopes C, Torres D, Oliveria A, Severo M, Alarcão V, Guiomar S et al. Inquérito Alimentar Nacional e de Atividade Física, IAN-AF 2015-2016: Relatório de Resultados. Universidade do Porto; 2017.

9. Sarafana S, Abecasis F, Tavares A, Soares I, Gomes A. Aleitamento Humano: evolução na última década. Acta Pediatr Port. 2006;37: 9-14.

10. Sandes AR, Nascimento C, Figueira F, Gouveira R, Valente S, Martins S et al. Aleitamento humano prevalência e factores condicionantes. Acta Med Port. 2007;20:193-200

11. Rocha LM, Gomes A. Prevalência do aleitamento humano nos primeiros seis meses de vida. Saúde Infantil. 1998;20:59-66.

12. Lanzaro C, Santos P, Guerra A, Hespanhol AP, Esteves MJ. Prevalência do Aleitamento Humano: comparação entre uma população Urbana e uma população Rural do Norte de Portugal. Acta Pediatr Port. 2015;46:101-8.

13. Kislaya I, Braz P, Dias C, Loureiro I. A evolução do aleitamento humano em Portugal nas últimas duas décadas: dados dos Inquéritos Nacionais de Saúde (1995/96 -1998/99-2005/06-2014). Lisboa: Instituto Nacional de Saúde Ricardo Jorge/Escola Nacional de Saúde Pública; 2017. Disponível em:http://www.cisp.ensp.unl.pt/.

14. Hobbs A, Mannion C, McDonald S, Brochway M, Suzanne C. The impact of caesarean section on breastfeeding initiation, duration and difficulties in the first four months postpartum. BMC Pregnancy and Childbirth. 2016; 16:90.

15. Cohen S, Alexander D, Krebs N, Young B, Cabana M, Erdmann P et al. Factors Associated with Breastfeeding Initiation and Continuation: A Meta-Analysis. The Journal of Pediatrics. 2018; 203,190-6.

16. Santacruz-Salas C, Aranda-Reneo I, Segura-Fragoso A, Cobo-Cuenca A, LaredoAguilera J, Carmona-Torres J. Mothers Expectations and Factors Influencing Exclusive Breastfeeding during the First 6 Months. Int J Environ Res Public Health. 2020; 17,77. 17. UNICEF/WHO: Innocent Declaration on the protection, promotion and support of breastfeeding. Florence: UNICEF and WHO; 1990.

18. World Health Organization, UNICEF. Baby-Friendly Hospital Initiative: Revised, updated and expanded for integrated care. 2009. https://apps.who.int/iris/ handle/10665/43593.

19. Philipp B, Merewood A, Miller L, Chawla N, Murphy-Smith M, Gomes J et al. Baby-friendly Hospital Initiative improves breastfeeding initiation rates in a US hospital setting. Pediatrics. 2001; 108:677-681.

20. Levy L, Bértolo H. Manual de aleitamento humano. Lisboa: Comité Português para a UNICEF / Comissão Nacional - Iniciativa Hospitais Amigos dos Bebés; 2012. 